\title{
Optimizing delivery of HIV testing services to strengthen the continuum of care
}

Project SOAR

Follow this and additional works at: https://knowledgecommons.popcouncil.org/departments_sbsr-hiv

Part of the Health Services Research Commons How does access to this work benefit you? Let us know!

\section{Recommended Citation}

Project SOAR. 2021. "Optimizing delivery of HIV testing services to strengthen the continuum of care," Learnings from Project SOAR, Synthesis Brief. Washington, DC: Population Council. 


\section{Optimizing Delivery of HIV Testing Services to Strengthen the Continuum of Care}

\section{Learnings from Project SOAR}

HIV testing is a critical entry point to life-saving antiretroviral therapy (ART) for those diagnosed with HIV and to prevention services for those at highrisk of acquiring HIV. Despite advances in testing, there remain a variety of social and programmatic challenges that prevent access to HIV testing services (HTS), particularly for men and key populations. HIV program managers and policymakers are increasingly invested in understanding how best to deliver HTS to different populations and maximize health system resources at facility and community levels.

Project SOAR recognizes the key role that HTS plays in achieving UNAIDS' 90-90-90 goals for controlling the HIV epidemic through testing, treatment, and viral suppression by 2020. Drawing on its extensive portfolio, Project SOAR has contributed to the evidence base and catalyzed research use by examining HIV testing among key populations, which also can inform strategies to help other populations to initiate and remain in care, reach viral suppression, and achieve positive health outcomes.

This brief synthesizes results from 12 studies across 10 countries (see map on next page for additional detail) that examined:

- Barriers to and enablers of HIV testing uptake

- Strategies for increasing uptake, optimizing delivery, and improving quality of HTS at the following service-delivery levels:

- Individual

- Community

- Facility

- Laboratory

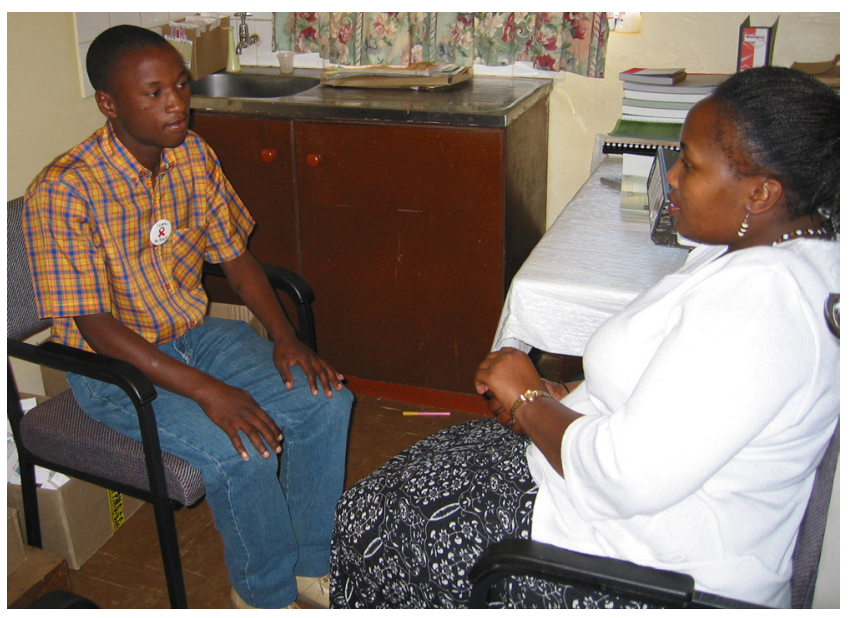

\section{KEY RECOMMENDATIONS}

Expand context-specific testing strategies at multiple levels of the health system. Based on SOAR research to date, these include:

1. HIV self-testing options to promote agency, awareness, and self-care, especially among key populations.

2. Integrated, community-based interventions, including DREAMS, to increase testing among adolescent girls and young women and their partners.

3. Enhanced facility-based services that leverage participatory approaches to identify and address HIV testing bottlenecks and gaps facing adult clients. Prioritize re-testing of HIV-negative pregnant and postpartum women to reach infants.

4. Rigorous quality management in HIV laboratory testing to avoid misclassification of results and unnecessary delays in care. 
Project SOAR HIV testing-related activities

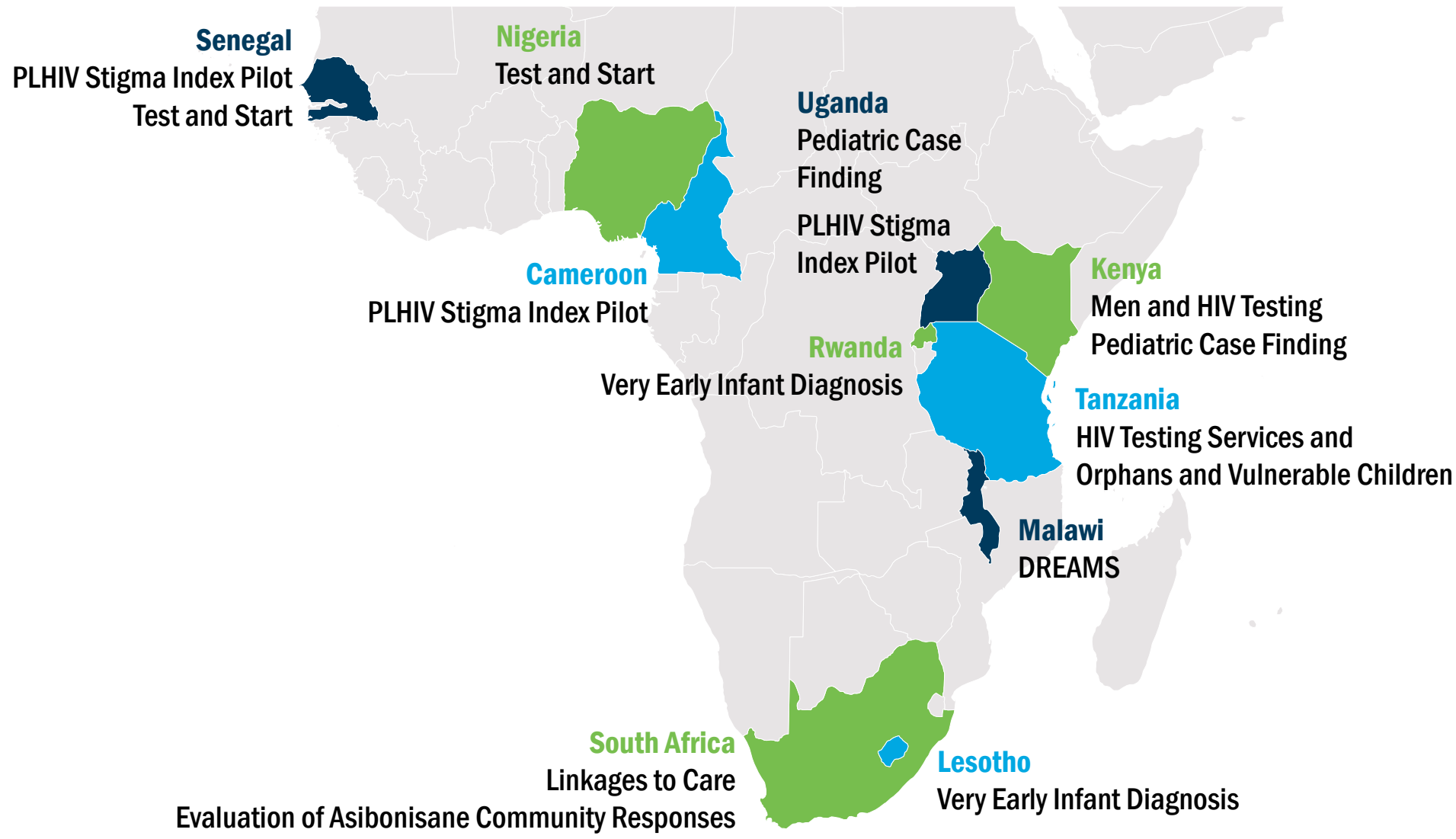

\section{ABOUT}

Project SOAR (Supporting Operational AIDS Research), was a 6-year USAID-funded implementation science initiative with over 70 activities in 21 countries. The Population Council led Project SOAR in collaboration with Avenir Health, Elizabeth Glaser Pediatric AIDS Foundation (EGPAF), Johns Hopkins University, Palladium, and The University of North Carolina at Chapel Hill.

\section{Research Utilization}

Project SOAR implemented a systematic and proactive research utilization (RU) approach to advance translation of research results into evidence-informed practice. This $\mathrm{RU}$ approach supported inclusion of end data users in the research process from inception to dissemination and fostered data-driven policy and program change.

\section{Series}

"Learnings from Project SOAR" seeks to highlight key results, recommendations, and examples of research impact across this 6-year, 21-country initiative.

The series includes five thematic briefs focused on biomedical prevention interventions, HIV testing services, HIV treatment strategies, stigma, and research utilization and capacity strengthening.

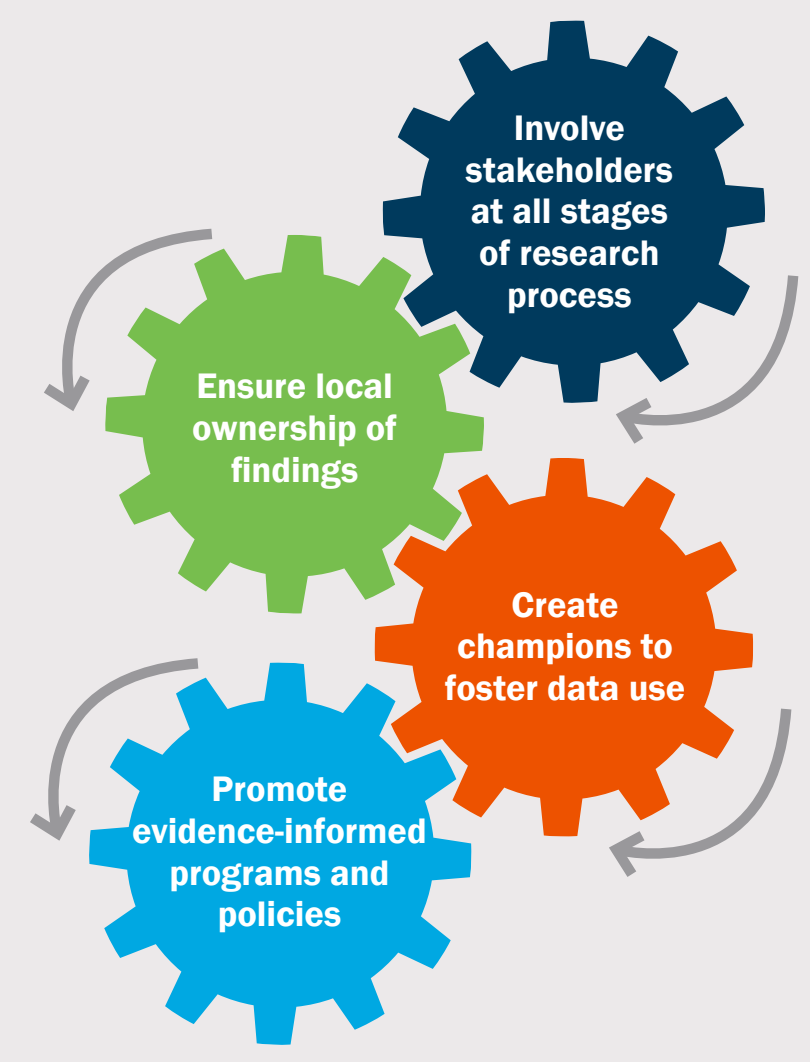

2 | Optimizing delivery of HIV testing services to strengthen the continuum of care 


\section{BARRIERS TO AND ENABLERS OF HIV TESTING UPTAKE}

Overview: To design effective programs and reduce disparities in uptake of HIV testing, country-specific data are needed regarding facilitators and barriers to HIV testing. SOAR examined factors influencing HIV testing in Cameroon, Kenya, Malawi, Senegal, South Africa, and Uganda and through surveys and interviews with providers and patients.

Results Summary: There remain critical individual-level barriers to testing due to stigma and fear, as well as facilitylevel barriers such as lack of privacy and confidentiality, distance, lack of integrated services, and missed provider opportunities for testing. Key enablers promoting testing among patients included trust in providers, quality of services, and flexible facility hours. Continued community- and country-level research efforts can help to support tailored and evidencebased interventions.

\section{Key findings}

In South Africa, interviews with patients, clinicians, and counselors revealed a variety of staff and client challenges to facility-based HIV testing.

To gain a richer understanding of the barriers to and opportunities for HTS from different perspectives, SOAR researchers conducted 22 in-depth interviews with clinic patients, clinicians, and HTS counselors. Despite spending more time with patients and having a greater role within HTS than a nurse or a doctor, HTS counselors often felt undervalued and unmotivated, and struggled with poorly defined roles, limited renumeration and acknowledgement, and lack of adequate workspaces. Clinicians mentioned huge workloads and clients' refusal to accept HTS. It was also noted that in only selecting suspected HIV cases and waiting until the end of consultations to offer HTS, they routinely missed multiple opportunities

Figure 1 Testing disparities in South Africa, Malawi, and Kenya

Women who tested in the past 12 months

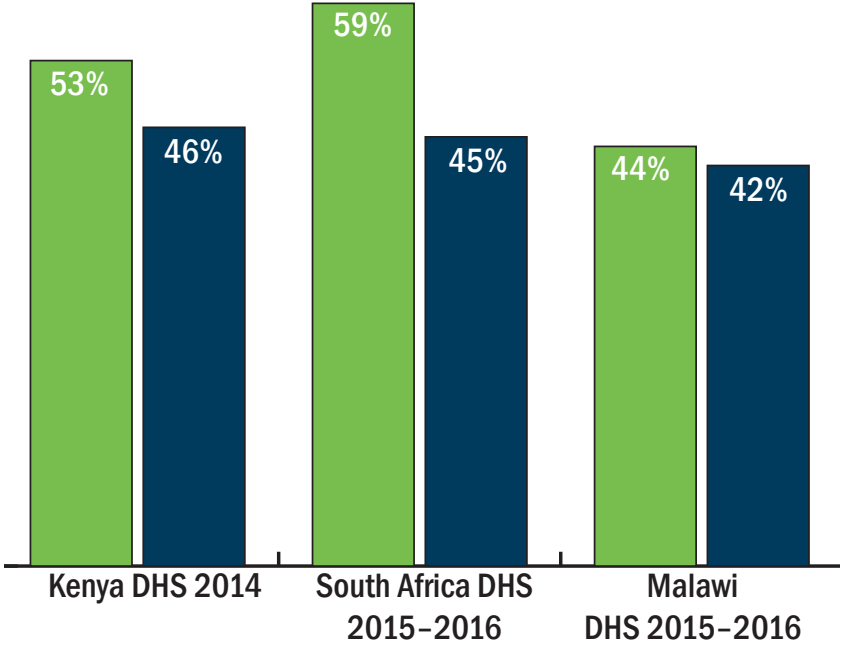

Kenya National Bureau of Statistics et al. 2016. Kenya Demographic and Health Survey 2014. Nairobi, Kenya and Rockville, Maryland, USA: Kenya National Bureau of Statistics and ICF.

National Department of Health (NDoH), Statistics South Africa (Stats SA), South African Medical Research Council (SAMRC), and ICF. 2019. South Africa Demographic and Health Survey 2016. Pretoria, South Africa, and Rockville, Maryland, USA: NDoH, Stats SA, SAMRC, and ICF.

National Statistical Office (NSO) [Malawil and ICF. 2017. Malawi Demographic and Health Survey 2015-16. Zomba, Malawi, and Rockville, Maryland, USA. NSO and ICF.

for testing. Clinic patients noted lack of privacy and confidentiality, fear of knowing one's HIV status, and long queues and additional waiting times of nearly one hour as barriers to testing. ${ }^{1}$

In Malawi and Kenya, male partners of adolescent girls and young women (AGYW) also identified multiple barriers to testing but were motivated to seek care due to trust in providers, quality of services, and flexible hours.

In Malawi, SOAR researchers conducted focus group discussions with 157 male partners of AGYW and indepth interviews with 49 men living with HIV to learn how to better engage men in HIV services. Similarly, in Kenya, researchers interviewed men who had sought HIV testing and men living with HIV to learn more about their perceived barriers to HIV testing and linkage to care. Study participants in both countries identified stigma and fear of testing positive as barriers to HIV testing, and also reported low HIV risk perception. Facility-related barriers included long wait times that disrupted livelihoods, lack of privacy and confidentiality, and distance to health services. ${ }^{2}$ 
In the Kenya study, men accessing HTS identified several factors that motivated them to seek services. These included having high risk perception; being ill; awareness of partner's status; having trust in the privacy, confidentiality, and quality of services; and flexible/extended operating hours. ${ }^{3}$

In Cameroon, Senegal, and Uganda, findings from a pilot of the updated People Living with HIV (PLHIV) Stigma Index 2.0 indicated that stigma remains a critical barrier to testing.

In 2017, Project SOAR researchers updated and tested the draft Stigma Index 2.0 (in collaboration with the original developers) in three countries: Cameroon, Senegal, and Uganda. Respondents were recruited from PLHIV networks, community-based organizations serving key populations, ART clinics, and through snowball sampling. A total of 1,207 PLHIV (about 400 in each country) participated in the pilot study. The majority of participants were in their mid-30s, female, and had known their HIV status for more than six years. Survey data reveal that delaying getting tested for HIV because of fears about how other people would respond if they tested positive ranged from 23 percent in Cameroon to a high of 41 percent in Uganda, indicating that stigma remains an important barrier to testing. ${ }^{4}$

In Nigeria, SOAR found suboptimal uptake of clinic-based testing among key populations, though testing was more common among men who have sex with men (MSM) and female sex workers (FSWs) recruited through outreach.

Researchers in Nigeria enrolled 605 untreated HIV-positive participants comprising 342 FSWs and 263 MSM in a cohort study in $2017 / 2018$. While national guidance recommends that key populations be tested every three months, more than a fourth of FSWs and MSM had never been tested for HIV prior to enrollment. Recent HIV testing was more common among FSWs and MSM recruited through outreach than through the clinic, suggesting the important role outreach can play in meeting the country's testing guidelines.

\section{STRATEGIES FOR INCREASING UPTAKE, OPTIMIZING DELIVERY, AND IMPROVING QUALITY OF HTS}

Overview: While there exist a variety of

avenues for HIV testing and initiation of care,

these often remain underutilized by many

potentially vulnerable communities, including

infants, orphans and vulnerable children (OVC),

AGYW, men, MSM, and FSWs. SOAR examined

strategies for improving quality HTS delivery

to these populations at different levels of care

in Kenya, Lesotho, Malawi, Nigeria, Rwanda,

Senegal, South Africa, Tanzania, and Uganda.

Results Summary:

Individual level-Self-testing is an acceptable approach to reach key populations that face barriers to engaging with routine outlets.

Community-based-Integrated, communitybased approaches are an effective way to improve testing among AGYW and their partners.

Facility-based services-Birth testing, screening tools for OVC, and enhanced ART delivery among pregnant and post-partum women can improve HIV testing and treatment initiation among infants and children. Systems-level approaches have the potential to increase rates of testing, including among men.

Optimizing testing resources-Screening tools, standardization of testing guidelines, and rigorous quality assurance measures for lab testing can reduce misdiagnoses and delays in care. 


\section{Key findings: Individual-level}

In Senegal, HIV self-testing (HIVST) was effective in reaching undiagnosed key populations.

In Senegal, SOAR used venue and social network approaches to distribute HIVST kits to key populations, including MSM and FSWs. A total of 1,839 HIVST kits were distributed in 2017/2018 and recipients participated in the pre- and post-test questionnaires. Use and acceptability of HIVST was high: 94 percent of post-test recipients used the kit, the vast majority within two days. Distribution of kits reached a high proportion of first-time testers, including about half of MSM who participated in the follow-up survey. About 3 percent had a positive result, many of whom were firsttime testers, but only nine who tested positive went for confirmatory testing. In addition, none with an invalid test result went for follow-up testing. The results from this study demonstrate that HIVST provides a complementary approach to reach populations who may face barriers to engagement with existing and routine HTS, including first-time testers (Figure 2). The study also highlights the importance of leveraging existing networks for distribution of HIVST kits and the need for active follow-up to support confirmatory testing. ${ }^{5}$

\section{Figure 2 HIV self-testing in Senegal}

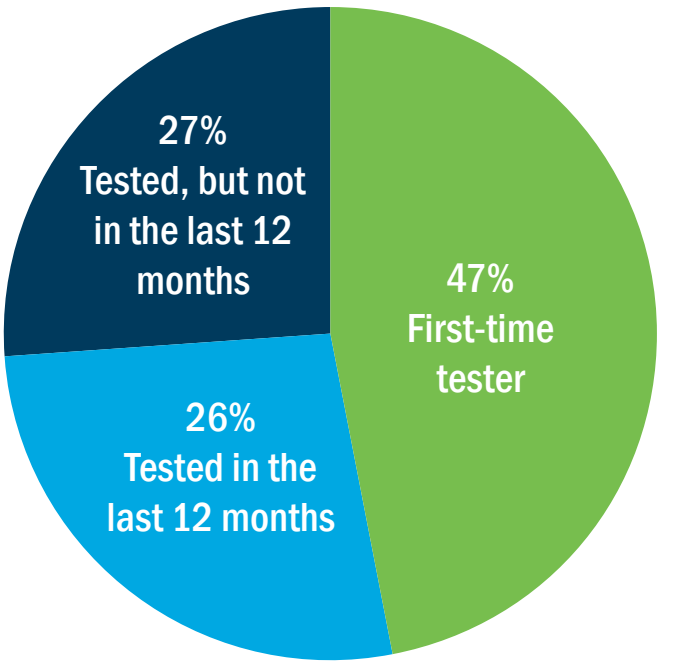

\section{Key findings: Community-based}

\section{In Malawi, an integrated, community-based program for AGYW was associated with an increase in HIV testing and knowledge of part- ner's HIV status.}

Project SOAR assessed the extent to which the DREAMS (Determined, Resilient, Empowered AIDSfree, Mentored, and Safe) initiative ${ }^{a}$ in Malawi contributed to reducing HIV risk among AGYW and their partners. A prospective cohort study was conducted with out-of-school AGYW (age 15-24 years) participating in DREAMS programming. Surveys and qualitative interviewers were conducted to assess the effects of multi-component, community-based interventions on a range of outcomes, including positive sexual health behaviors, improved knowledge and skills to increase self-efficacy, and economic empowerment. AGYW in the cohort reported significantly higher rates of HIV testing in the last 12 months at endline. There was also a significant increase in knowledge of partner's HIV status. Of AGYW who knew their partner's HIV status at endline, 89 percent learned about it via couples' counseling and $\mathbf{8 3}$ percent of couples had been tested within the last year at a government health facility. Interviewed AGYW reported that they often tested with their partners, and all AGYW reported having a positive experience at the health facility. ${ }^{2}$

\section{In South Africa, a community-based program focused on urban informal settlements led to an increase in testing among women but struggled to reach men.}

SOAR researchers used a representative sample of 1,528 adult men (18-35) and women (18-24) from a longitudinal study to evaluate the Asibonisane Community Responses program implemented in urban informal settlements in KwaZulu-Natal, South Africa. The program was designed to promote uptake of HIV services and transform gender norms to create

aln specific high HIV-risk communities, DREAMS delivered through the public and private sector, a comprehensive package of evidence-based, prevention strategies. These strategies went beyond the health sector to address the structural drivers that fuel AGYW's HIV risk, including poverty, gender inequality, sexual violence, social isolation, and limited schooling. 
an enabling environment for HIV prevention behaviors among adult men and women. Although 90 percent had been tested for HIV at some point in the past at baseline, a lower proportion had been tested in the last six months. Program participation led to a $\mathbf{1 7}$ percent increase in the probability of testing for women but had no effect on testing for men due, in part, to the fact that the program did not reach men who were least likely to be tested, such as men who had recently migrated to the community. ${ }^{6}$

\section{Key findings: Facility-based testing services}

\section{Post-partum}

In Lesotho and Rwanda, testing at birth was found to be acceptable and offered benefits to infants. However, countries need to consider costs and implementation feasibility before its adoption.

In Lesotho and Rwanda, SOAR researchers evaluated the feasibility, acceptability, and cost of diagnosing HIV at birth, utilizing a multi-component mixed methods study. Birth testing was acceptable to women, health workers, and laboratory staff, with most recommending rolling out birth testing nationally. The major benefit cited by women and health workers was earlier knowledge of the child's HIV status and therefore earlier initiation of treatment for positive children resulting in a reduction in infant mortality. Most women interviewed had no negative perceptions of very early infant diagnosis and generally expressed satisfaction with how services were delivered. While infants identified as infected at birth were initiated on treatment more quickly than those tested at six weeks, the turnaround time for receiving results was still long and varied between one and two months. In order to reduce turnaround time, it may be less burdensome and costly to strengthen counseling, tracing, and testing efforts beginning at six weeks of life rather than adding birth testing. ${ }^{7}$

\section{Pedatric}

In Kenya and Uganda, undetected HIV infection and failure to distribute ART to pregnant and breastfeeding women contributed to missed detection of HIV infection in children.

In Kenya and Uganda, SOAR investigated missed pediatric cases to better understand where and why cases were not identified earlier. Researchers assessed a total of 174 newly identified, HIV-positive children, ages $2-14$. Nearly 75 percent were diagnosed in outpatient departments and maternal and child health/prevention of mother-to-child transmission of HIV entry points. Undetected incident HIV infection and non-receipt of ART by mothers contributed to missed detection of infection in children. Two-thirds of the children's mothers tested in antenatal care were HIV negative, suggesting becoming infected later in the pregnancy or postpartum. Lack of re-testing of HIV-negative mothers during pregnancy and postpartum and failure to link newly diagnosed and known HIV-positive women to treatment are missed opportunities to identify pediatric cases. $^{8}$
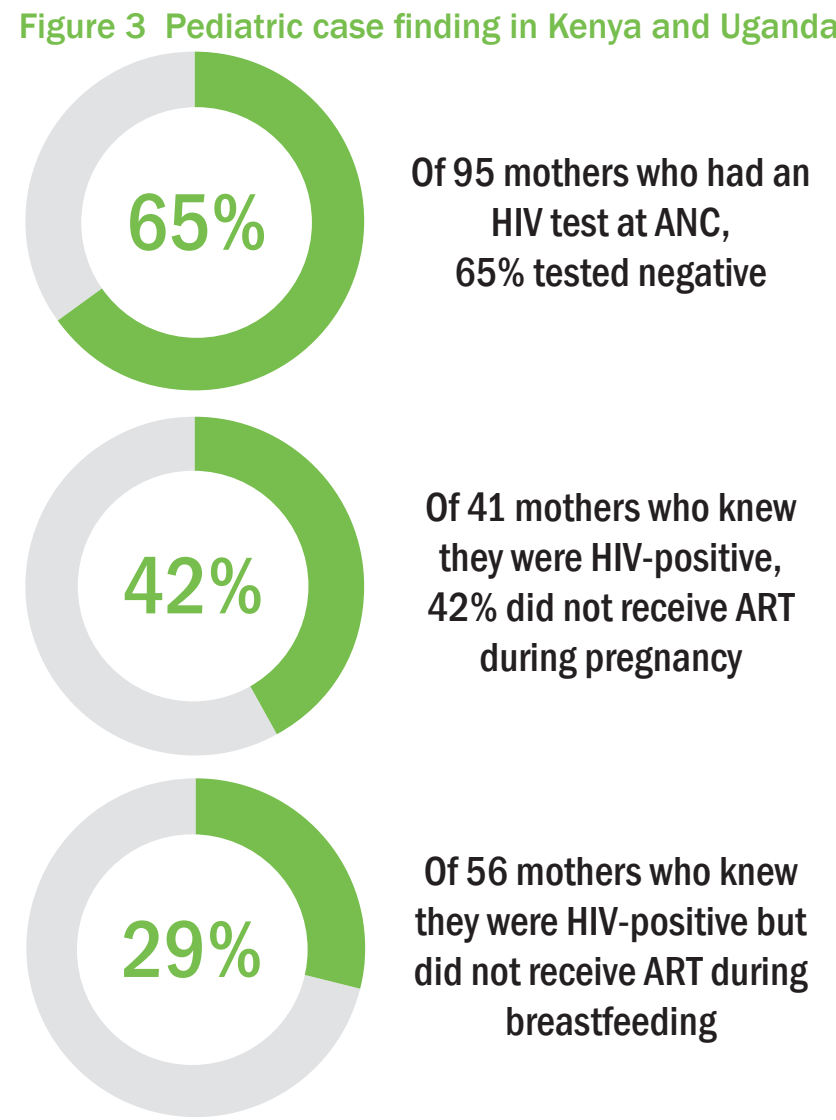

Of 56 mothers who knew they were HIV-positive but did not receive ART during breastfeeding 
Adult

In South Africa, a participatory, systems approach was found to increase uptake of facility-based HIV testing, successfully reaching men.

Project SOAR tested a systems approach to streamline facility-based HTS in 10 public health facilities in Gauteng province, South Africa. Before use of a systems approach, HTS was typically offered to patients after two or more hours of waiting in multiple queues. Based on formative data to identify gaps and bottlenecks, the clinics implemented a number of system-level changes, including promoting HTS daily in waiting areas, increasing recommendations of HTS by clinicians, shifting the time during a visit when HTS was recommended, and adjusting HTS counselor hours to increase availability and private counseling space. Comparing data from before and after implementation of the system changes, a considerably higher proportion of patients reported being offered testing. Among those who received testing, the absolute number of people tested increased, and proportion of male testers increased from 20 to 38 percent. The study highlights the role a systems approach can play in enhancing facilitybased HTS. Despite the improvements, the offering and uptake of HIV testing remained low overall. ${ }^{1}$

\section{Key findings: Optimizing testing resources}

\section{In Tanzania, a pediatric HIV risk assessment tool was feasible for screening children and adolescents in communities and facilities, but administration of screening questions was inconsistent in communities.}

Screening tools that identify children most vulnerable to HIV can help prioritize testing resources for those individuals. SOAR tested HIV risk screening tools in Tanzania among nearly 20,000 children and adolescents in communities and facilities. The optimal screening tool had five items: whether a relative had died, ever admitted to hospital, have a family member with HIV, have a cough, or was the adolescent sexually active (if over age 10). This short screening tool-for which those who had at least one of these characteristics was classified as eligible for testinghad a sensitivity of $\mathbf{8 5}$ percent, and specificity of
44 percent, as compared to participants' HIV test results. Community participants and community care workers who administered the screening tool found home-based HIV risk screening and testing to be acceptable, but fidelity to the screening questions was inconsistent, with community care workers often skipping questions. As HIV risk screening tools are scaled up in Tanzania and beyond, close monitoring of implementation is critical, as are cost-effectiveness analyses. $^{9,10}$

\section{In Nigeria, standardization of testing guide- lines and rigorous quality management of lab- oratory tests resulted in no misclassification of rapid test results.}

Misclassification of HIV status has been reported to occur due to false-positive diagnoses; in some settings, misclassifications have been substantial, carrying a heavy social and financial cost. SOAR assessed HIV-positive misclassification rates among newly diagnosed key and general population clients in a selection of programs in Nigeria where "test and treat" has been implemented. A total of 788 confirmatory blood samples were collected from newly diagnosed HIV-positive clients. In this study, there was no misclassification of rapid test results, confirming that only HIV-positive clients were placed on treatment. The perfect concordance between rapid HIV test and ELISA from all the sites may be attributable to the oversight of a HTS technical working group and the adherence of implementing partners to the standardized and rigorous quality assurance management system for both community and facility-based HIV testing services. ${ }^{11}$ 


\section{RESEARCH IMPACT SPOTLIGHT}

\section{Senegal Test and Start (TnS) Study Informs Large-scale Distribution of HIVST Kits in Senegal}

Project SOAR's Senegal TnS study demonstrated that expanding access to HIVST may increase HIV testing among key populations and can effectively engage first-time testers. As a result, the Ministry of Health integrated HIVST in its Test and Treat Guidelines. Based on its experience with HIVST distribution under the SOAR study, partner Enda Santé is also leading a large-scale distribution of more than 30,000 HIVST kits in Senegal to key populations and their partners, as part of the regional ATLAS project. Led by the nongovernmental organization Solthis and funded by UNITAID, this project incorporates self-testing protocols from SOAR and is operating in three countries (Côte d'Ivoire, Mali, and Senegal) over a three-year period (2018-2021).

\section{REFERENCES}

1. Hoffmann, Christopher J. and Tonderai Maputo. 2020. "Can a systems approach increase facility-based HIV testing and accelerate linkage to care for those who test positive? Findings from South Africa," Project SOAR Final Report. Washington, D.C.: Population Council.

2. Pilgrim, Nanlesta et al. 2020. "Evidence to support HIV prevention for adolescent girls and young women (AGYW) \& their male partners: results from Malawi Dreams studies with AGYW, male partners of AGYW, men living with HIV, and program implementing partners," Project SOAR Final Report. Washington, D.C.: Population Council.

3. Okal, Jerry D. et al. 2020. "'It is always better for a man to know his HIV status'-A qualitative study exploring the context, barriers and facilitators of HIV testing among men in Nairobi, Kenya," PLoS ONE 15(4): e0231645. doi: 10.1371/journal.pone.0231645

4. Project SOAR. 2018. "The People Living with HIV Stigma Index 2.0: Results from pilot testing in three countries," Project SOAR Results Brief. Washington, D.C.: Population Council.

5. Lyons, Carrie E. et al. 2019. "Use and acceptability of HIV self-testing among first-time testers at risk for HIV in Senegal," AIDS and Behavior 23(Suppl 2): 130-141. doi: 10.1007/s10461-019-02552-2

6. Psaki, Stephanie et al. "What are we learning about HIV testing in informal settlements in KwaZulu-Natal, South Africa?," PLoS ONE, under review.

7. Gill, Michelle et al. 2018. "Piloting very early infant diagnosis of HIV in Lesotho: Acceptability and feasibility among mothers, health workers and laboratory personnel," PLoS ONE 13(2):e0190874. doi: 10.1371/ journal.pone.0190874
8. Gill, Michelle M., Rose Masaba, and Eliab K. Natumanya. 2020. "Pediatric HIV active case finding in Kenya and Uganda: strategies and linkage to treatment," Project SOAR Final Report. Washington, D.C.: Population Council.

9. Gill, Michelle M. and Gretchen Antelman. 2020. "Use of an HIV risk screening tool in pediatric community and facility settings in Tanzania," Project SOAR Final Report. Washington, D.C.: Population Council.

10. Gill, M. M. et al. 2021. "HIV risk screening and HIV testing among orphans and vulnerable children in community settings in Tanzania: Acceptability and fidelity to lay-cadre administration of the screening tool," PLoS One 16(3): e0248751. doi: 10.1371/journal.pone.0248751

11. Project SOAR. 2019. "No misclassification of HIV-positive diagnoses: good news for Nigeria's 'test and treat' policy," Project SOAR Results Brief. Washington, D.C.: Population Council.

Suggested citation: Project SOAR. 2021. "Optimizing delivery of HIV testing services to strengthen the continuum of care," Learnings from Project SOAR Synthesis Brief. Washington, D.C.: Population Council.

For more information, please visit Project SOAR.
Project SOAR was a six-year (September 2014-January 2021) cooperative agreement funded by the United States President's Emergency Plan for AIDS Relief and the U. S. Agency for International Development (Agreement No. AID-OAA-A-14-00060). The contents of this brief are the sole responsibility of Project SOAR and Population Council and do not necessarily reflect the views of PEPFAR, USAID, or the United States Government.

Population Council led the Project SOAR consortium in collaboration with Avenir Health, Elizabeth Glaser Pediatric AIDS Foundation, the Johns Hopkins University, Palladium, and The University of North Carolina at Chapel Hill.
Project SOAR/Population Council

4301 Connecticut Avenue, NW, Suite 280

Washington, DC 20008

Tel: +12022379400

e-mail: ProjectSOAR@popcouncil.org projsoar.org

()Population Council, April 2021

Cover photo credit: Population Council 